\title{
Los sistemas de producción caprina en el municipio de Molagavita, sobre la cuenca del río Chicamocha, Colombia
}

\author{
Javier Enrique Vargas-Bayona*, MVZ, César Augusto Serrano-Novoa, MV, \\ Ph.D., Daniel Adyro Martínez-Bello, MV, MSc. ${ }_{2,5}$, Guadalupe Rodríguez- \\ Galván, MVZ, MSc. ${ }_{3}$, Lourdes Zaragoza-Martínez, MVZ, Ph.D., Lady \\ Katerine Serrano-Mujica, MVZ, MSc. $(c)_{4}$ \\ , Estudiante de Maestría en Ciencias en Producción Agropecuaria Tropical, Universidad Autónoma \\ de Chiapas, Chiapas, México \\ ${ }_{2}$ Grupo de Investigación en Ciencias Animales, Universidad Cooperativa de Colombia, Bucaramanga, Colombia \\ ${ }_{3}$ Grupo de Investigación Agricultura Familiar (AGRIF), Instituto de Estudios Indígenas, Universidad Autónoma \\ de Chiapas, Chiapas, México \\ ${ }_{4}$ Estudiante de Maestría en Farmacología, Universidad Federal de Santa María, Santa María, Brasil \\ ${ }_{5}$ Becario Colciencias, candidato a Doctor en Estadística y Optimización, Universidad de Valencia, Valencia, España
}

Recibido: 11 de junio del 2015 Aprobado: 12 de noviembre del 2015

Autor de correspondencia: Javier Enrique Vargas-Bayona. Facultad de Veterinaria y Zootecnia, Universidad Cooperativa de Colombia, Bucaramanga, Santander, Colombia. Calle 30A n. ${ }^{\circ}$ 33-51. Teléfono: (577) 6458500, ext. 7095. Correo electrónico: javier.vargasb@campusucc.edu.co

Cómo citar este artículo: Vargas-Bayona JE, Serrano-Novoa CA, Martínez-Bello DA, Rodríguez-Galván G, Zaragoza-Martínez L, Serrano-Mujica LK. Los sistemas de producción caprina en el municipio de Molagavita, sobre la cuenca del río Chicamocha, Colombia. Spei Domus. 2015;11(23): 9-16. doi: http://dx.doi.org/10.16925/sp.v11i23.1362

Resumen. Introducción: el municipio de Molagavita (Colombia) hace parte de la cuenca del río Chicamocha, donde se desarrollan actividades agrícolas y ganaderas, principalmente la cría de caprinos. El objetivo de la investigación fue identificar las características correspondientes a los sistemas productivos caprinos de Molagavita presentes sobre la cuenca del río Chicamocha. Materiales y métodos: se hizo un muestreo de bola de nieve; se encuestaron a seis productores. La encuesta estaba constituida por 10 parámetros con información sobre características generales, población, nutrición y alimentación animal, salud, reproducción, producción, genética, administración y economía y mercadeo; rescató la opinión del productor acerca del sistema de producción empleado y su satisfacción. Se usó Microsoft Office Excel $^{\circledR} 2010$ para su análisis, además se implementó un análisis descriptivo para las variables cuantitativas. Resultados: la posesión de la tierra es propia en el $100 \%$ y la extensión de las fincas es en promedio de $7 \pm 8,62$ ha. Con respecto a la infraestructura y a los equipos empleados, se cuenta con corral, saladeros y bebederos en el $100 \%$ de las granjas y con cerca eléctrica en el $50 \%$. El sistema de producción es extensivo en el $66 \%$, con orientación a la producción de carne $100 \%$. No se lleva ningún tipo de registro y al consultar sobre algunos parámetros zootécnicos, se reportaron promedios para peso al nacimiento de $2,75 \pm 0,69 \mathrm{~kg}$ y peso al beneficio de 29,5 9,35 kg. Conclusiones: la mayor actividad económica de la zona es la comercialización de cabritos, aves de corral y productos agrícolas.

Palabras clave: conservación, desarrollo rural, economía campesina, ganado caprino. 


\title{
Goat Production Systems in the Municipality of Molagavita on the Chicamocha River Basin, Colombia
}

\begin{abstract}
Introduction: The municipality of Molagavita (Colombia) is part of the Chicamocha river basin, where farming and livestock activities are carried out, especially goat breeding. The aim of the research was to identify the characteristics of Molagavita goat production systems present on the Chicamocha river basin. Materials and methods: Snowball sampling was conducted; six producers were surveyed. The survey consisted of 10 parameters with information on general characteristics, population, animal nutrition and feeding, health, reproduction, production, genetics, management, and economics and marketing; the opinion of producers on the production system used and their satisfaction was obtained. Microsoft Office Excel $^{\circledR} 2010$ was used for analysis, and a descriptive analysis for quantitative variables was implemented. Results: $100 \%$ own their land and extension of farms is $7 \pm 8.62$ ha on average. With regard to infrastructure and equipment used, there are corrals, salting facilities, and troughs in $100 \%$ of farms and electric fence in $50 \%$. The production system is extensive in $66 \%$, and oriented towards meat production in $100 \%$. No record is kept and, when finding out about some zootechnical parameters, averages for birth weight of $2.75 \pm 0.69$ $\mathrm{kg}$ and benefit weight $29.5 \pm 9.35 \mathrm{~kg}$ were reported. Conclusions: The main economic activity in the area is the marketing of goats, poultry, and agricultural products.
\end{abstract}

Keywords: conservation, rural development, rural economy, goats.

\section{Os sistemas de produção caprina de Molagavita, sobre a bacia do rio Chicamocha, Colômbia}

Resumo. Introdução: o município de Molagavita (Colômbia) faz parte da bacia do rio Chicamocha, onde se desenvolvem atividades agrícolas e pecuárias, principalmente a criação de cabras. O objetivo desta pesquisa foi identificar as características correspondentes aos sistemas produtivos caprinos de Molagavita presentes sobre a bacia do rio Chicamocha. Materiais e métodos: fez-se uma amostragem em bola de neve; realizou-se uma enquete com seis produtores. Esta estava constituída por 10 parâmetros com informação das características gerais, população, nutrição e alimentação animal, saúde, reprodução, produção, genética, administração, economia e marketing; resgatou a opinião do produtor ao respeito do sistema de produção empregado e sua satisfação. Usou-se o Microsoft Office Excel ${ }^{\circ}$ 2010 para a análise; além disso, foi implementada uma análise descritiva para as variáveis quantitativas. Resultados: a posse da terra é própria em 100\%, e a extensão das fazendas é em média de $7 \pm 8,62$ ha. Sobre a infraestrutura e os equipamentos utilizados, conta-se com um curral, saleiros e bebedouros em $100 \%$ das granjas e com cerca elétrica em $50 \%$. O sistema de produção é extensivo em $66 \%$, com orientação à produção de carne em $100 \%$. Não se encontrou nenhum tipo de registro e, ao consultar sobre alguns parâmetros zootécnicos, relataram-se médias para peso no nascimento de $2,75 \pm 0,69 \mathrm{~kg}$ e peso no abate de $29,5 \pm 9,35 \mathrm{~kg}$. Conclusões: a maior atividade econômica da região é a comercialização de cabritos, aves de criação e produtos agrícolas.

Palavras-chave: conservação, desenvolvimento rural, economia camponesa, gado caprino. 


\section{Introducción}

El municipio de Molagavita es uno de los 13 municipios que conforman la provincia de García Rovira, en el departamento de Santander. Su extensión territorial es de 19.600 ha, divididas políticamente en un casco urbano y en 17 veredas que cuentan con 3320 predios rurales, que ocupan el $97 \%$ del total del área del municipio. Las principales actividades económicas son la agricultura y la cría de animales, desarrolladas en altitudes que van desde los 720 hasta los 3000 msnm. La producción caprina se desarrolla en las veredas Chicacuta, Vega Infante y Toma de Agua, en los sectores Mesetas y Río Chico [1]. Según Avendaño [2], las vegas del río Chicamocha son terrenos fértiles destinados para la agricultura. Las cabras son llevadas a la zona de ladera donde se defienden por sí solas entre cactus de diferentes variedades, pringamoza (Urtica urens), guasabara (Cylindropuntia spp), leguminosas arbóreas y otras plantas propias del bosque seco tropical. Para el 2012, la Alcaldía Municipal de Molagavita [3] reportó en su inventario de población caprina un estimado de 6800 cabezas. La cría de ganado caprino ha crecido, lo cual ha despertado nuevo interés y ha generado la necesidad de profundizar conocimientos en este tipo de producción y, con más razón, en la cabra santandereana. Esta especie es considerada como un recurso zoogenético que se ha formado y ha tenido un gran desarrollo y adaptabilidad a las condiciones del medio hostil del bosque seco del cañón del Chicamocha.

La caprinocultura forma parte de la tradición de las comunidades, y de acuerdo con las características topográficas del cañón del Chicamocha, se hace imposible que en el ecosistema se desarrollen grandes hatos de ganado bovino y grandes extensiones agrícolas. Lo anterior ha promovido que la caprinocultura se posicione como el renglón más importante de la economía campesina, pero son escasos los estudios técnicos sobre dicha actividad en la región. Este trabajo se desarrolló con el objetivo de generar información que permita conocer las características de los sistemas de producción caprina presentes sobre la cuenca del río Chicamocha en el municipio de Molagavita, Santander, Colombia.

\section{Materiales y métodos}

El estudio se llevó a cabo en el municipio de Molagavita (veredas Chicacuta y Toma de Agua, sector Las Mesetas y Río Chico). Se hizo un muestreo mediante la técnica bola de nieve [4], empleando como herramienta una encuesta directa a seis productores, conformada por 10 parámetros: características generales, población, nutrición y alimentación animal, salud, reproducción, producción, genética, administración, y economía y mercadeo, siguiendo el modelo propuesto por varios autores $[5,6,7]$. Como complemento se le hizo una entrevista semiestructurada al $30 \%$ de la población encuestada, y entrevistas abiertas a informantes clave como comercializadores y técnicos, entre otros [8]. Se elaboró un análisis descriptivo para las variables cuantitativas y se utilizaron tablas de contingencia.

\section{Resultados}

A continuación, se presentarán los datos que arrojó cada una de las preguntas formuladas en la encuesta, que se relacionan con las principales características del sistema de producción que predomina en el municipio de Molagavita. Estos resultados obtenidos se basan en la opinión de cada uno de los productores encuestados, quienes accedieron previo consentimiento informado y amparados bajo la Ley 1581 de 2012 y el Decreto 1377 de 2013, mediante los cuales se dictan disposiciones generales para la protección de datos personales en la República de Colombia.

Aspectos generales. En la vereda Toma de Agua, sector Las Mesetas, se encontró que las fincas tienen un promedio de $7 \pm 8,62$ ha de extensión y que la tenencia de la tierra es propia, mientras que en el sector de Río Chico las tierras y los animales están en comodato. En la vereda Chicacuta, en el sector del caserío, las casas son propias y las cabras se crían en terrenos de ladera que son comunales. El $100 \%$ de los predios dispone de alguna fuente de agua, ya sea proveniente de acueducto, río o nacimiento hídrico en el predio. Con respecto a la infraestructura y a los equipos empleados, se cuenta con corral, saladeros y bebederos en el $100 \%$ y con cerca eléctrica en el $50 \%$.

La edad promedio del productor fue de $47,4 \pm 8,8$ años, la experiencia en la actividad caprina registrada fue de 30,3 $\pm 19,5$ años y el nivel de escolaridad fue educación básica primaria en el $100 \%$ de los productores. El 66,66\% reportó que recibía asistencia técnica de las autoridades municipales en temas 
como producción y reproducción, nutrición y alimentación animal, dejando a un lado la genética y la administración.

Nutrición y alimentación. El 66,66 \% de las cabras pastorea en sistemas extensivos y el $32,33 \%$, en pastoreo semi-intensivo. Como complemento alimenticio, el 33,33\% ofrece melaza a las cabras; algunos productores afirman que en la temporada seca les proporcionan a las cabras subproductos de los residuos de cosecha; como fuente de minerales, el 83,33\% suministra sal común y el 16,66\%, sal mineralizada. Como oferta forrajera en las laderas, los productores citaron las siguientes especies de plantas: cují (Prosopis juliflora), mataratón (Glieriridia sepium), espino gallinero (Pithecellobium dulce), orégano u oreganillo (Borreria anthospermoides), cacho de cabra (Popomax totuosa), espino blanco o uña de cabra (Acacia farnesiana) y guásimo (Guazuma ulmifolia).

Población animal y genética. Los grupos raciales predominantes son: la cabra de biotipo santandereana, las razas nubiana y boer, y los respectivos cruzamientos que están orientados a la producción de carne; se destaca un potencial genético uniforme para la mayoría de las fincas donde se crían cabras. Según los productores entrevistados, se tienen algunas cabras de temperamento lechero, que se ordeñan cuando se encierra a los animales y se usa la leche para el autoconsumo. Además de la cría de caprinos, se encontraron otras especies de animales; en el total de las fincas encuestadas tenían aves de corral, caninos, felinos y bovinos.

Producción y reproducción. En cuanto a los parámetros zootécnicos reportados en la encuesta, se destacan la edad al primer parto a los $11 \pm 0,89$ meses; el intervalo entre partos es de 5,66 $\pm 0,57$ meses. Debido a las características del sistema, los productores no tienen en cuenta la condición corporal de las hembras y no registran los días al primer servicio posparto. El sistema de monta es natural en el $100 \%$ de los casos, y de acuerdo con estos datos reportados, se puede deducir que para el primer servicio no se tiene en cuenta la edad y el peso de las hembras. El peso de las crías al nacimiento fue de $2,75 \pm 0,69 \mathrm{~kg}$, la edad al destete fue de $3,16 \pm 0,49$ meses con un peso de $9,6 \pm 3,69 \mathrm{~kg}$, $\mathrm{y}$ el peso al beneficio fue de $21,8 \pm 7,98 \mathrm{~kg}$ a una edad de $11,2 \pm 4,60$ meses. El número de hembras por macho en promedio es de $22,92 \pm 10,71$. El criterio de descarte para machos y hembras es la edad. Los partos se concentran en los meses de junio-julio y diciembre-enero, y no se acompaña al momento. Otros parámetros se pueden apreciar en la tabla 1 .

Salud. El mayor problema de tipo sanitario que afecta a las cabras en Molagavita es la miasis cavitaria, generada por la mosca de la cabeza (Oestrus ovis); le siguen los síndromes diarreicos que afectan sobre todo a la población lactante y en crecimiento. Según los productores encuestados, las mordeduras de murciélago (Desmodus rotundus)

Tabla 1. Descriptivo de los parámetros productivos y reproductivos en los sistemas de producción caprina en el municipio de Molagavita, según los productores encuestados

\begin{tabular}{|c|c|c|c|c|c|c|}
\hline Parámetro & $\mathbf{n}$ & Media & CV \% & Mediana & Máximo & Mínimo \\
\hline Peso al nacimiento & 6 & $2,75 \pm 0,75$ & 27,57 & 2,75 & 4 & 2 \\
\hline Edad al destete & 6 & $3,16 \pm 0,40$ & 12,89 & 3 & 4 & 3 \\
\hline Peso al destete & 5 & $9,6 \pm 3,64$ & 37,98 & 10 & 15 & 5 \\
\hline Edad al beneficio & 5 & $11,2 \pm 4,60$ & 41,11 & 10 & 16 & 8 \\
\hline Peso al beneficio & 6 & $24,16 \pm 6,911$ & 28,59 & 21,5 & 35 & 17 \\
\hline Peso de venta en pie & 5 & $21,8 \pm 7,98$ & 36,61 & 25 & 30 & 9 \\
\hline Edad al primer servicio & 6 & $6 \pm 0,89$ & 14,9 & 6 & 7 & 5 \\
\hline Edad al primer parto & 6 & $11 \pm 0,89$ & 8,13 & 11 & 12 & 10 \\
\hline Intervalos entre partos & 3 & $5,66 \pm 0,57$ & 10,18 & 6 & 6 & 5 \\
\hline Número de hembras/macho & 6 & $19 \pm 13,05$ & 68,7 & 13,5 & 40 & 7 \\
\hline Vida útil de las hembras & 4 & $5,75 \pm 2,98$ & 51,93 & 5 & 10 & 3 \\
\hline Vida útil de los machos & 3 & $4,66 \pm 4,61$ & 98,97 & 2 & 10 & 2 \\
\hline
\end{tabular}

Fuente: elaboración propia 
son menores y lo atribuyen a las pocas cavernas o cuevas que hay en las laderas. En el 33,33\% de los predios, se hacen tratamientos contra ectoparásitos y endoparásitos. Según los productores, dos veces al año la Administración Municipal hace programas de desparasitación para rumiantes y caninos. En su gran mayoría, los productores consultan al médico veterinario cuando se les presenta un problema sanitario en los rebaños.

Economía y mercadeo. Al consultar sobre los aspectos económicos, se reporta que solo el $50 \%$ de los productores maneja un cuaderno de registro de ingresos; en general, no se maneja un sistema contable para la producción pecuaria y agrícola. La comercialización de caprinos y carnes es baja, pues el 66,66 \% de los productores destina las carnes para el autoconsumo; en el año se venden algunos cabritos en pie de acuerdo con las necesidades de las familias, pero la mayor venta se concentra en el mes de junio cuando se celebran las ferias y fiestas del municipio, y en los meses de diciembre y enero se vende la carne en canal a $\$ 7000$ pesos (USD $\$ 1=2550$ COP). El estiércol, o caprinaza, es destinado como fuente de abono en los cultivos.

Administración. En los sistemas de producción caprina, no se implementa ningún tipo de estrategias de planeación u organigramas para tener un objetivo claro y organizado del sistema productivo; el $100 \%$ de los predios no implementa registros reproductivos, productivos, ni sanitarios. El $50 \%$ no utiliza un sistema de identificación de animales, y el porcentaje restante los identifica mediante muescas o señales en las orejas y algunas orejeras.

\section{Discusión}

Aspectos generales. El área promedio es de 7 ha, resultado inferior a los reportados por Atuesta et al. [9] para los sistemas de producción caprina en Barichara, Santander (292,2 ha). La variación del tamaño de las fincas se atribuye a que los productores de Molagavita solo tienen en cuenta las áreas destinadas para las labores agrícolas, las cabras pastorean en zonas con pendientes de casi el $70 \%$ y en muchos casos son terrenos comunales que no son aptos para cultivos. La cría de caprinos es una actividad que se considera ejercida en grandes extensiones de tierra por pequeños productores. Sin embargo, Roncallo et al. [10] consideran que el tamaño de la propiedad rural debe tomarse con reserva al momento de catalogar a un productor como pequeño o grande según la zona agroecológica donde está ubicada.

Según Torres [11] y Guerrero et al. [12], los sistemas de producción caprina de la cuenca del Chicamocha cuentan con instalaciones muy escasas; el corral se limita solo para encierro de los animales sin distinción; son elaborados con materiales de la región como madera, varas, rocas, techos en láminas de zinc o teja y pisos en tierra; como comederos y bebederos se usan rocas calizas de forma plana o hueca.

Para la variable de edad de los productores, Rebollar-Rebollar et al. [13] y García [14] reportaron un rango entre 45 y 56 años en los estados de México y Guerrero (México), muy similar al reportado en este trabajo.

Población animal y genética. El alto porcentaje de utilización de la cabra biotipo santandereana por los productores encuestados se da porque no hay un grado de especialización tecnológica y productiva. Al igual que lo que reportaron Gall y Reule [6], y Vargas-Bayona et al. [15], es frecuente encontrar ejemplares mestizos (principalmente santandereana/anglo-nubiana y santandereana/alpina) y razas de tipo lechero como la alpina, la saanen y la toggenburg. Las cabras pastorean de forma libre en los municipios de Cepita, Capitanejo, Los Santos y Molagavita, y recorren distancias desde 1 hasta 20 $\mathrm{km}$, en altitudes desde 100 hasta $700 \mathrm{~m}$ [6].

Entre los animales de ganadería están, en mayor porcentaje, las aves de corral, seguidas por los bovinos, y como animal de compañía, los caninos. La integración de otras especies de animales domésticos con la producción caprina forma parte de la cultura del productor [16]. La composición de diferentes especies animales en las granjas permite complementar la producción del rebaño con la venta de los otros animales [17].

Producción y reproducción. El seguimiento que hacen los criadores a los parámetros productivos es deficiente; el control de peso de los animales se hace con báscula, pero en las fincas donde no hay herramientas para el pesaje, el productor pone en práctica su experiencia y conocimiento tácito al calcular el peso de los animales "a ojo".

El peso al nacer de las crías fue de 2,75 $\pm 0,75 \mathrm{~kg}$. Estos reportes son similares a los de Moreno [18] en sistemas de producción orientados a la genética y a 
la leche en el trópico alto, donde los pesos reportados fueron de 2,61 $\pm 0,84 \mathrm{~kg}$. El destete, por lo general, lo hacen las madres separando a las crías; varios autores recomiendan que el destete se dé entre $10 \mathrm{y}$ 120 días, tiempo en el que los cabritos realizan un consumo regular de alimentos forrajeros, o cuando las crías ya tengan 2,5 veces más peso que su peso de nacimiento [19]. El peso al destete muestra la influencia de la madre en los rebaños donde las crías reciben alimento natural, lo que demuestra la habilidad materna [20].

Los resultados obtenidos muestran una tendencia a beneficiar y comercializar animales jóvenes. Hacer el beneficio con cabritos hasta los 12 meses de edad favorece el alcance en la regularidad de la oferta de las crías cebadas [21].

De acuerdo con los sistemas de manejo descritos antes, no se tiene control ni criterio alguno para el primer servicio de las hembras jóvenes; la monta natural no controlada es característica del sistema de producción extensivo $[6,10]$. La relación de hembras está entre lo recomendado por la Empresa Brasileira de Pesquisa Agropecuária Embrapa, usar 30 hembras por macho en sistemas con monta no controlada [22].

Según los productores encuestados, los partos ocurren durante todo el año. Sin embargo, se hace evidente que hay dos periodos con mayor frecuencia que el resto del año. La mayoría ocurre de noviembre a enero y de junio a agosto. Esto es igual a lo reportado por Gall y Reule [6], quienes afirman que durante los meses de junio a septiembre y de enero a marzo se presentan las más altas tasas de concepción, lo que significa que los periodos de mayor actividad reproductiva se dan durante las temporadas secas.

El intervalo entre partos (IEP) es un parámetro muy importante para evaluar la eficiencia reproductiva y productiva del rebaño, y se relaciona con los objetivos de producción, manejo reproductivo, raza, edad, número de partos, tipo de parto (simple, doble o triple), la alimentación, la época del año, el destino de las crías y el efecto macho [23, 24]. En el nororiente colombiano, Gall y Reule [6] encontraron IEP de 9,07 $\pm 0,23$ meses sin diferenciar el objeto productivo. Maia y Costa [23] señalaron un IEP de 8 meses en cabras para producción de carne, y para las razas lecheras el IEP varía de 10 a 12 meses de acuerdo con los tiempos de la lactancia.

Salud. Los problemas sanitarios suelen ser diferentes en cada país e incluso en las regiones, al igual que los procedimientos para el control.
En México, Vargas [25] reportó como problemas sanitarios más frecuentes en las cabras: intoxicación por el consumo de plantas tóxicas, parásitos internos y externos, ectima contagioso y problemas respiratorios. Los problemas de mordedura de murciélago son menores a los que se presentan en los municipios de Jordán [26] y Aratoca [27].

La población más susceptible a enfermedades según los productores encuestados son los animales en lactancia, un resultado similar a lo reportado por Moreno [18]. Esta condición probablemente se debe al poco manejo que se hace a las hembras en el momento del parto, a la edad del primer parto o al poco peso que tienen las crías al nacer.

Economía y mercadeo. Estimar los costos de producción y estudiar la viabilidad económica es muy importante para el desarrollo de las actividades pecuarias y la caracterización de un sistema de producción, y es también el primer paso para una evaluación económica [28].

Ninguno de los productores encuestados ha hecho estimaciones económicas de balances, ni estados de pérdidas y ganancias. Los registros económicos son más frecuentes en sistemas de producción especializados, cuyo objetivo es la venta de leche o derivados lácteos, o de animales para el pie de cría con un alto valor en genético $[18,29]$.

Según varios autores, los productores agropecuarios con un bajo nivel tecnológico no conocen los reales costos de la producción, pero sí es frecuente que ellos lleven los registros de ingresos. Esto se debe a que los productores integran la producción caprina con la agrícola; así, juegan un papel importante en la mitigación de la pobreza y en el mejoramiento de la seguridad alimentaria en los hogares, lo que permite que los productores generen ingresos de la venta de excedentes de leche $y$ de animales vivos o en canal [30,31].

La finalidad productiva de las granjas caprinas es principalmente el autoconsumo y la venta de carne. Una parte de la producción se canaliza en el autoconsumo, que se hace a diario, en fiestas religiosas, en reuniones familiares, en festividades de fin de año y en otras actividades de carácter social. El alto porcentaje de autoconsumo es un indicador de que la orientación comercial y empresarial es baja.

Administración. El manejo tradicional de las cabras ha hecho que los productores no reconozcan su producción como una empresa. El $100 \%$ de los encuestados no cuenta con organigrama, ni planes de mejoramiento del sistema de producción. 
Estos resultados coinciden con lo reportado por Valerio [5] en sistemas de producción caprina en República Dominicana, en los cuales no se cuenta con registros productivos, reproductivos, sanitarios y económicos que permitan hacer un análisis de la eficiencia y la viabilidad de la actividad. En sistemas con alto grado de especialización donde se reportan registros de producción, reproducción, costos y ventas, el productor puede analizar los indicadores para mejorar los objetivos de producción de pie de cría y leche [28].

\section{Conclusiones}

En los sistemas de producción caprina en el municipio de Molagavita, la cabra es considerada como una fuente de ahorro y de ingresos económicos ante una necesidad. Las cabras son una alternativa de producción sobre todo en terrenos que no tienen otros usos, y se convierten en una fuente de proteína para la alimentación de las familias.

El alto porcentaje del biotipo de la cabra santandereana, presente en las fincas, es un indicador de preservación y conservación del recurso zoogenético ejercido por los productores. Pero la introducción de otras razas, sin criterio alguno, pone en riesgo la desaparición del biotipo santandereana.

\section{Agradecimientos}

Los autores agradecen a los caprinocultores del municipio de Molagavita, Santander, por su valiosa colaboración.

\section{Referencias}

[1] Alcaldía Municipal de Molagavita. Sitio oficial de Molagavita en Santander, Colombia [Internet]. 2015 [citado 2015 junio 2]. Disponible en: http://www. molagavita-santander.gov.co/index.shtm

[2] Avendaño RA. El cañón del Chicmocha. Bucaramanga: Sic Editorial Ltda.; 2009.

[3] Alcaldía Municipal de Molagavita. Plan de Desarrollo 2012-2015 "Por un Mejor Futuro" [Internet]. Molagavita: Alcaldía Municipal de Molagavita; 2012 [citado 2015 junio 2]. Disponible en: http:// www.molagavita-santander.gov.co/apc-aa-fi- les/34363938656662363339333137633964/plan-desarrollo-2012-2015-molagavita.pdf

[4] Wayne WD. Bioestadística. Base para el análisis de las ciencias de la salud. 4. ${ }^{a}$ ed. México: Limusa Wiley; 2010.

[5] Valerio D. Análisis de competitividad del sistema ovino y caprino del noroeste de la República Dominicana [tesis doctoral]. Córdoba: Universidad de Córdoba, España; 2009.

[6] Gall C, Reule M. Producción caprina en Colombia. Informe Técnico N. ${ }^{\circ}$ 6. Bogotá: Instituto Colombiano Agropecuario; 1989.

[7] Grajales HA, Ospina OF, Manrique C, Téllez G, Vásquez R, Roncallo B, et al. Desarrollo e implementación de un Sistema de Gestión Tecnológica en los Sistemas de Producción de la Cadena Ovino-Caprina para el mejoramiento de su competitividad - SIGETEC. Bogotá: Ministerio de Agricultura y Desarrollo Rural, Universidad Nacional de Colombia, Universidad de la Salle y Corpoica; 2007.

[8] Rodríguez G, Sanabria N, Ramírez C, Guevara F, Perezgrovas $\mathrm{R}$, Zaragoza L. La gallina de rancho y el caldo de gallina como elementos de identidad campesina frailescana. Actas Iberoamericanas de Conservación Animal. 2012;(2):25-34.

[9] Atuesta MF, Daza LM, Del Río FA, Garnica YM, Martínez DA, Serrano-Novoa CA, et al. Caracterización de los sistemas productivos caprinos en el municipio de Villanueva, Santander. Actas Iberoamericanas de Conservación Animal. 2012;(2):293-96.

[10] Roncallo B, Toloza A, Barros J, Silva J, Araújo A, Mejía M. Sistemas de producción ovino-caprinos en los departamentos de La Guajira, Cesar y Magdalena. Corpoica, senA, editores. Agustín Codazzi: Corpoica; 1999.

[11] Torres J. Caracterización de la raza caprina criolla santandereana. Trabajo de grado para optar al titulo de Zootecnista. Bucaramanga: Universidad Nacional Abierta y a Distancia UnAD, Facultad de Ciencias Agrarias, Programa de Zootecnia; 2002.

[12] Guerrero LM, Téllez JN, Vera M, Garza BA, Rincón JL, Martínez-Bello DA, et al. Caracterización de los sistemas de producción caprinos presentes en el municipio de Jordán, Santander, Colombia. Actas Iberoamericanas de Conservación Animal. 2012;2:171-4.

[13] Rebollar-Rebollar S, Hernández-Martínez J, Rojo-Rubio R, Guzmán-Soria E. Gastos e ingresos en la actividad caprina extensiva en México. Agronomía Mesoamericana. 2012;23(1):159-65.

[14] García M. La producción de cabras en la cañada de Huamuxtitlán, estado de Guerrero [tesis de maestría]. Puebla: Colegio de Postgraduados; 2013. 
[15] Vargas-Bayona JE, Serrano-Novoa CA, Martínez-Bello DA, Rodríguez G, Zaragoza L. Los sistemas productivos caprinos en la provincia de Guanentá, Santander, sobre la cuenca del río Chicamocha. Actas Iberoamericanas de Conservación Animal. 2014;(4):237-9.

[16] Alexandre G, González-García E, Lallo CH, Ortega-Jimenez E, Pariacote F, Archimède H, et al. Goat management and systems of production: global framework and study cases in the Caribbean. Small Ruminant Research. 2010;89(2):193-206.

[17] Gaspar P, Escribano AJ, Mesías FJ, Escribano M, Pulido AF. Goat systems of Villuercas-Ibores area in sw Spain: problems and perspectives of tradicional farming systems. Small Ruminal Research. 2011;97(1):1-11.

[18] Moreno D. Nivel de desarrollo tecnológico de los sistemas de producción ovinos y caprinos en las regiones Centro, Norte y Valles Interandinos de Colombia [tesis de maestría]. Bogotá: Universidad Nacional de Colombia; 2013.

[19] Ciffoni EM. Estudo fenotípico e genético do peso ao nascimento e ao desmame de caprinos Saanen na região de Curitiba, estado do Paraná, Brasil. Arquivos de Ciências Veterinárias e Zoologia da UNIPAR. 1999;2(1):43-52.

[20] Lemos JO. Seleção e melhoramento genético aplicados ao gado zebu no Brasil. O Zebu do Brasil. 1988;126(17):47-50.

[21] Andrade JJ. Caracterização da caprinocultura nas microrregiões da Chapada do Apodi e de Angicos do estado do Rio Grande do Norte [tesis de maestría]. Macaíba: Universidade Federal do Rio Grande do Norte; 2013.

[22] Empresa Brasileira de Pesquisa Agropecuária, Embrapa. Criação de caprinos e ovinos. Cartilha. Brasilia DF: Empresa Brasileira de Pesquisa Agropecuária, Embrapa Caprinos; 2007. Report N. ${ }^{\circ}$ 978-85-7383-419-2.

[23] Maia M, Costa AN. Estro e atividade ovariana pós-parto em cabras Canindé, associados ao manejo da amamentação. Revista Brasileira de Reprodução Animal. 1998;22(1):35-43.
[24] Tenório CG. Avaliação do efeito macho sobre o desempenho reprodutivo de cabras da raça Anglo-Nubiana criadas no semiárido do estado de Pernambuco [tesis doctoral]. Recife: Universidade Federal Rural de Pernambuco; 2011.

[25] Vargas S. Análisis y desarrollo del sistema de producción agrosilvopastoril caprino para carne en condiciones de subsistencia de Puebla, México [tesis doctoral]. Córdoba: Universidad de Córdoba, España; 2003.

[26] Guerrero LM, Téllez JN, Vera M, Garza BA, Rincón JL, Martínez-Bello DA, et al. Caracterización de los sistemas de producción caprinos presentes en el municipio de Jordán, Santander, Colombia. Actas Iberoamericanas de Conservación Animal. 2012;2:171-4.

[27] Vargas-Bayona JE, Serrano-Novoa CA, Martínez-Bello DA, Rodríguez G, Zaragoza L. Caracterización de los sistemas de producción caprina en el municipio de Aratoca, Santander sobre la cuenca del río Chicamocha. Actas Iberoamericanas de Conservación Animal. 2014;4:234-6.

[28] Stivari TS, Monteiro AL, Gameiro A, Chen R, Silva C, Paula EF, et al. Viabilidade econômico-financeira de sistemas de produção de cordeiros não desmamados em pastagem com suplementação em cocho ou pasto privativo. Revista Brasileira de Saúde e Produção Animal. 2013;14(3):396-405.

[29] Pezzoti G, Velásquez R, Polanco D. Una aproximación a la caracterización técnico-económica de los sistemas de producción caprinos lecheros cercanos al Valle de Aburrá. Revista Colombiana de Ciencias Pecuarias. 2011;24(3):404.

[30] Al-Assaf AA. Economic implications of small ruminant diseases in the northern area of Jordan. Journal of Food, Agriculture and Environment. 2012;10(1):323-6.

[31] Al-Khaza’leh J, Reiber C, Al Baqain R, Valle Zárate A. A comparative economic analysis of goat production systems in Jordan with an emphasis on water use. Livestock Research for Rural Development. 2015;27(5). Disponible en: http://www.lrrd.org/lrrd27/5/khaz27081.html 